\title{
PROPRIEDADES FÍSICAS DE BIODIESEL DE PINHÃO MANSO: AVALIAÇÃO DE MÉTODOS PREDITIVOS PARA CÁLCULOS DE VISCOSIDADE E DENSIDADE.
}

\author{
P. M. FLORIDO ${ }^{1}$, K. K. ARACAVA ${ }^{1}$, I. C. F. MORAES ${ }^{1}$, R. A. FERRARI ${ }^{2}$, P. J. A. SOBRAL ${ }^{1}$ e C. \\ B. GONÇALVES ${ }^{1}$ \\ ${ }^{1}$ Universidade de São Paulo, Departamento de Engenharia de Alimentos \\ ${ }^{2}$ Instituto de Tecnologia de Alimentos, Centro de Ciência e Qualidade de Alimentos \\ E-mail para contato: cintiabg@usp.br
}

\begin{abstract}
RESUMO - O conhecimento de propriedades físicas, como viscosidade e densidade, permite o aprimoramento das etapas envolvidas na produção de biodiesel. Devido à inviabilidade de aquisição desses dados em todas as condições de interesse, métodos para estimá-las têm grande importância prática. $\mathrm{O}$ objetivo deste trabalho foi testar diferentes modelos para o cálculo de viscosidade e densidade de biodiesel de pinhão manso. As viscosidades foram obtidas em um reômetro rotacional AR 2000 (TA Instruments), e as densidades em um densímetro digital DMA4500 (Anton Paar). Para as predições, foram testadas a Regra de Kay (tanto para a viscosidade como para a densidade) e os modelos de contribuição de grupos GC-UNIMOD (viscosidade) e GC-VOL (densidade). Apesar da complexidade dos modelos de contribuição de grupos, estes apresentaram desempenho muito parecido com o dos modelos mais simples. Para o cálculo da viscosidade, os desvios médios relativos (DMR) foram ao redor de $7 \%$, e para a densidade, os valores de DMR ficaram em torno de $0,7 \%$.
\end{abstract}

\section{INTRODUÇÃO}

O crescente esgotamento das reservas de combustíveis fósseis do mundo tem despertado o interesse pela busca de fontes alternativas de energia, especialmente de combustíveis renováveis como o biodiesel. Este é obtido através da transesterificação de óleos ou gorduras, tanto de origem animal como vegetal. A composição em metal-ésteres de um determinado biodiesel afeta diretamente a viscosidade, que pode possuir uma grande variação, dependendo da fonte oleaginosa (Allen et al., 1999). Dentre as fontes oleaginosas não comestíveis, o pinhão manso (Jatropha curcas) tem sido identificado como uma das mais proeminentes com potencial para a produção de biodiesel (Choudhurya et al., 2014).

A viscosidade é, certamente, uma das propriedades de transporte mais significantes do biodiesel, pois tem efeito direto nos coeficientes de transferência de calor e massa, afetando o desempenho do motor (Ceriani et al., 2007). Sabe-se também que esta propriedade é altamente influenciada pela composição em metil ésteres e pela temperatura. Por esta razão, a viscosidade cinemática $(v)$ é utilizada como padrão regulador para biodiesel. Esta propriedade está relacionada 


\section{9 a 22 de outubro de 2014 \\ Florianópolis/SC}

com a viscosidade dinâmica $(\eta)$ através da densidade $(\rho)$, de acordo com a Equação 1.

$$
v=\frac{\eta}{\rho}
$$

A literatura reporta vários dados de viscosidade para biodiesel (Pratas et al., 2011, entre outros) ou misturas de ésteres metílicos (Gonçalves et al., 2007). Porém, apesar da simplicidade dos métodos de determinação de viscosidade, nem sempre é viável medi-la em todas as condições de interesse. Conseqüentemente, métodos para estimar estas propriedades em diferentes temperaturas e concentrações são de grande importância prática. Dentre os modelos existentes na literatura, destacam-se os baseados na fração molar, como a Regra de Kay (Azian et al., 2001), tanto na forma simples como modificada. Dentre os modelos baseados no conceito de contribuição de grupos, tem-se o CG-UNIMOD (Cao et al., 1993), para a viscosisade, e o GCVOL (Elbro et al., 1991), para a densidade.

Desta forma, o objetivo deste trabalho foi determinar dados experimentais de densidade e viscosidade de biodiesel de pinhão manso, e testar os modelos simples e complexos supramencionados para o cálculo destas propriedades físicas.

\section{MATERIAL E MÉTODOS}

\subsection{Produção do biodiesel de pinhão manso}

A reação de transesterificação foi realizada em um reator encamisado de $10 \mathrm{~L}$, no qual reagiram a $45^{\circ} \mathrm{C}$, óleo bruto de pinhão (obtido por prensagem das sementes), metanol e metilato de sódio $30 \%$, como catalisador, conforme descrito em Ferrari et al.(2005). Obteve-se então o biodiesel e como coproduto a glicerina. $\mathrm{O}$ biodiesel separado por decantação foi neutralizado por sucessivas lavagens com solução de $\mathrm{HCl}$ 0,5\%. A água de lavagem foi separada por decantação e os resíduos de umidade foram retirados por filtração com sulfato de sódio anidro.

Na determinação da composição em ésteres de ácidos graxos presente no biodiesel foi feita a transmetilação, realizada de acordo com o método de Hartman \& Lago (1973), usando solução de cloreto de amônia e ácido sulfúrico em metanol como agente esterificante.

A cromatografia gasosa foi realizada em um cromatógrafo a gás, equipado com amostrador automático; injetor split, razão 75:1; coluna capilar CP-SIL 88 (100 m x 0,25 mm i.d., 0,20 mm de filme); detector por ionização em chama (FID) e uma workstation para aquisição dos dados. A temperatura da coluna foi programada com uma temperatura inicial de $120^{\circ} \mathrm{C}$ mantida por $2 \mathrm{~min}$, seguida por aquecimento de $120^{\circ} \mathrm{C}$ a $220^{\circ} \mathrm{C}$ a uma taxa de $2,2^{\circ} \mathrm{C} / \mathrm{min}$ e de $220^{\circ} \mathrm{C}$ a $235^{\circ} \mathrm{C}$, a taxa de $1,5^{\circ} \mathrm{C} / \mathrm{min}$, permanecendo a $235^{\circ} \mathrm{C}$ por 15 minutos. $\mathrm{O}$ Hidrogênio foi usado como gás de arraste na vazão de $1 \mathrm{~mL} / \mathrm{min}$ e o gás "make-up" (Nitrogênio) a $30 \mathrm{~mL} / \mathrm{min}$. A temperatura do injetor foi de $270^{\circ} \mathrm{C}$ e a temperatura do detector, $310^{\circ} \mathrm{C}$. $\mathrm{O}$ volume de injeção foi de $1 \mathrm{~mL}$. A identificação dos 
ácidos graxos foi realizada através da comparação do tempo de retenção dos ácidos graxos das amostras e padrões e co-cromatografia. A quantificação foi realizada por normalização de área e os resultados foram expressos em $\mathrm{g} / 100 \mathrm{~g}$ de amostra.

\subsection{Determinação das propriedades físicas}

Os dados de densidade foram determinados em um Densímetro Digital de bancada (DMA 4500, Anton Paar). As amostras de biodiesel foram injetadas diretamente no equipamento com o auxílio de uma seringa descartável, sem bico rosqueado. Após a estabilização da temperatura, o equipamento forneceu o resultado direto da densidade. Todas as medidas de densidade foram realizadas em triplicata. As viscosidades foram determinadas em triplicata com o auxílio de um reômetro rotacional AR 2000 (TA Instruments), com geometria de cilindros de parede dupla. Todas as medidas de propriedades físicas foram obtidas em 6 diferentes temperaturas: $10,20,30,40,50$ e $60{ }^{\circ} \mathrm{C}$.

\subsection{Estimativa das propriedades físicas}

Os dados experimentais das propriedades físicas dois tipos de modelos foram utilizados para testar a capacidade de alguns modelos da literatura. $O$ primeiro deles considera o biodiesel como uma mistura de ésteres metílicos e inclui uma aproximação linear (Regra de Kay Simples) e sua forma logarítmica (Regra de Kay Modificada), apresentadas nas Equações 2 e 3, respectivamente. A Regra de Kay simples foi utilizada para a estimativa tanto das densidades como das viscosidades do biodiesel nas diferentes temperaturas. Já a Regra de Kay Modificada foi aplicada apenas para o cálculo das viscosidades.

$$
\begin{aligned}
& P_{\text {mistura }}=\sum_{i=1}^{n} x_{i} \cdot\left(P_{i}\right) \\
& \ln (v)_{\text {mistura }}=\sum_{i=1}^{n} x_{i} \cdot \ln \left(v_{i}\right)
\end{aligned}
$$

Na Equação 2, o termo $P$ representa a propriedade física, densidade $(\rho)$ ou viscosidade cinemática $(v)$ e $x_{i}$, o qual aparece tanto na Equação 2 como na 3, representa a fração molar de cada éster metílico $i$ na mistura (biodiesel) contendo $n$ compostos.

Como pode ser observado, os modelos supramencionados (Equações 2 e 3) requerem o conhecimento da densidade e da viscosidade de cada composto formador da mistura. Neste caso, foram utilizados os dados obtidos por Pratas et al. (2010) e Pratas et al. (2011).

O segundo tipo de modelo estudado, GCVOL (Elbro et al., 1991) para a densidade e GCUNIMOD (Cao et al., 1993) para a viscosidade, são baseados no conceito de contribuição de grupos, capazes de estimar as propriedades de uma mistura, tratando-a como uma solução de grupos funcionais das moléculas que as constituem. Sendo assim, cada propriedade física ou química pode ser considerada como a soma das contribuições feitas por cada um destes grupos e por suas 
interações. Esta propriedade aditiva, característica dos métodos de contribuição de grupos, é a grande vantagem dos modelos baseados neste conceito, pois a quantidade de informações necessárias para determinar as propriedades de uma mistura qualquer pode ser bem menor do que se fossem consideradas as contribuições de cada um de seus possíveis componentes. De fato, o número de grupos funcionais é bem menor comparado ao número de moléculas de interesse neste trabalho.

Para a utilização dos modelos baseados no conceito de contribuição de grupos, é necessário o conhecimento dos parâmetros de interação entre os grupos funcionais presentes nas moléculas da mistura de interesse. Tanto para o GCVOL (Elbro et al., 1991; Ihmels et al., 2002) como para o GCUNIMOD (Cao et al., 1993), a literatura já apresenta um vasto número de parâmetros de interação entre vários pares de grupos funcionais, incluindo os que constituem os ésteres metílicos: $\mathrm{CH}_{3}, \mathrm{CH}_{2}$, $\mathrm{CH}=\mathrm{e} \mathrm{CH}_{2} \mathrm{COO}$.

O modelo GCVOL (Elbro et al., 1991) é representado pela Equação 4 a seguir:

$$
\rho=\frac{\sum_{j} x_{j} M_{j}}{\sum_{j} x_{j} V_{j}}
$$

Na Equação 4, $x, M$ e $V$ são, respectivamente, a fração molar, a massa molar e o volume molar do componente $j$. $V_{j}$ pode ser obtido pela Equação 5, na qual $n_{i}$ é o número de grupos $i$ e $\Delta v_{i}$ é a dependência da temperatura $(T)$, dada pela função polinomial apresentada na Equação 6, na qual os parâmetros $A_{i}, B_{i}$ e $C_{i}$ foram obtidos de Ihmels e Gmehling (2002).

$$
\begin{aligned}
& V=\sum_{i} n_{i} \Delta v_{i} \\
& \Delta v_{i}=A_{i}+B_{i} T+C_{i} T^{2}
\end{aligned}
$$

O GC-UNIMOD (Group-Contribution Thermodynamics-Viscosity Model) é semelhante ao método UNIFAC proposto para predição do equilíbrio de fases, que leva em consideração duas contribuições para a viscosidade cinemática da mistura ( $\left.v_{\text {mistura }}\right)$ : uma parte combinatorial e outra residual, como mostra a Equação 7.

$$
\ln \left(v_{\text {mistura }}\right)=\sum_{i=1}^{n}\left[\xi_{i}^{C}+\xi_{i}^{R}\right]
$$

A parte combinatorial, $\xi_{i}^{C}$, considera as diferenças no tamanho das moléculas presentes na mistura, de modo que somente as propriedades das substâncias puras são necessárias para o cálculo. O termo residual, $\xi_{i}^{R}$, é mais complexa e leva em consideração a energia de interação entre os pares de grupos funcionais presentes na mistura. A completa descrição deste modelo pode ser encontrada em Gonçalves et al., 2007). Os valores de $R_{k}$ (parâmetro de volume do grupo $k$ ) e $Q_{k}$ (parâmetro de área do grupo $k$ ), presentes nos termos combinatorial e residual, respectivamente, foram obtidos de Fredenslund e Sorensen (1994). Os parâmetros de interação entre os grupos funcionais foram obtidos de Larsen et al. (1987). 


\section{RESULTADOS E DISCUSSÃO}

A Tabela 1 apresenta a composição em ésteres metílicos do biodiesel de pinhão manso, obtido segundo a metodologia apresentada na seção 2.1. Os dados estão apresentados em porcentagem mássica e porcentagem molar.

Tabela 1 - Composição em ésteres metílicos do biodiesel de pinhão manso

\begin{tabular}{|c|c|c|c|c|c|}
\hline $\begin{array}{c}\text { Metil éster de } \\
\text { ácido graxo }\end{array}$ & $\begin{array}{c}\text { Fórmula } \\
\text { molecular }\end{array}$ & Nome comum & $\begin{array}{c}\text { Massa molar } \\
\left(\mathrm{g} \cdot \mathrm{mol}^{-1}\right)\end{array}$ & $\begin{array}{c}\text { Fração mássica } \\
(\%)\end{array}$ & $\begin{array}{c}\text { Fração molar } \\
(\%)\end{array}$ \\
\hline $\mathrm{C} 16: 0$ & $\mathrm{C}_{18} \mathrm{H}_{36} \mathrm{O}_{2}$ & $\begin{array}{c}\text { Metil } \\
\text { palmitato }\end{array}$ & 284,48 & 15,58 & 16,78 \\
\hline $\mathrm{C} 16: 1$ & $\mathrm{C}_{18} \mathrm{H}_{34} \mathrm{O}_{2}$ & $\begin{array}{c}\text { Metil } \\
\text { palmitoleato }\end{array}$ & 282,46 & 0,85 & 0,92 \\
\hline $\mathrm{C} 18: 0$ & $\mathrm{C}_{20} \mathrm{H}_{40} \mathrm{O}_{2}$ & $\begin{array}{c}\text { Metil } \\
\text { estearato }\end{array}$ & 312,53 & 6,60 & 6,45 \\
\hline $\mathrm{C} 18: 1$ & $\mathrm{C}_{20} \mathrm{H}_{38} \mathrm{O}_{2}$ & Metil oleato & 310,51 & 41,92 & 41,19 \\
\hline $\mathrm{C} 18: 2$ & $\mathrm{C}_{20} \mathrm{H}_{36} \mathrm{O}_{2}$ & Metil linoleato & 308,50 & 34,66 & 34,30 \\
\hline $\mathrm{C} 18: 3$ & $\mathrm{C}_{20} \mathrm{H}_{34} \mathrm{O}_{2}$ & $\begin{array}{c}\text { Metil } \\
\text { linolenato }\end{array}$ & 306,48 & 0,18 & 0,18 \\
\hline $\mathrm{C} 20: 0$ & $\mathrm{C}_{22} \mathrm{H}_{44} \mathrm{O}_{2}$ & $\begin{array}{c}\text { Metil } \\
\text { araquidato }\end{array}$ & 340,58 & 0,20 & 0,18 \\
\hline
\end{tabular}

A Tabela 2 a seguir apresenta as densidades e as viscosidades cinemáticas do biodiesel de pinhão manso para todas as temperaturas estudadas.

Tabela 2 - Densidades $\left(\rho\right.$, em $\left.\mathrm{kg} \cdot \mathrm{m}^{-3}\right)$ e viscosidades cinemáticas $\left(\nu, \mathrm{em} \mathrm{mm} \cdot \mathrm{s}^{-2}\right)$ de biodiesel de pinhão manso em função da temperatura $\left(T\right.$, em $\left.{ }^{\circ} \mathrm{C}\right)$

\begin{tabular}{|c|c|c|}
\hline $\mathrm{T}\left({ }^{\circ} \mathrm{C}\right)$ & $\rho\left(\mathrm{kg} \cdot \mathrm{m}^{-3}\right)$ & $v\left(\mathrm{~mm}^{2} \cdot \mathrm{s}^{-1}\right)$ \\
\hline 10 & 889,70 & 10,7519 \\
\hline 20 & 882,52 & 8,0117 \\
\hline 30 & 875,36 & 6,1995 \\
\hline 40 & 868,20 & 4,9483 \\
\hline 50 & 860,99 & 4,0576 \\
\hline 60 & 853,71 & 3,4028 \\
\hline
\end{tabular}

De acordo com os dados experimentais apresentados na Tabela 2, é possível corroborar a diminuição dos valores das propriedades físicas frente ao aumento da temperatura. Pode-se observar também que o biocombustível obtido possui propriedades que estão de acordo com os padrões internacionais, que estabelecem faixas de viscosidade cinemática a $40{ }^{\circ} \mathrm{C}$ entre $3,5-5,0 \mathrm{~mm}^{2} \cdot \mathrm{s}^{-1}$, no caso da regulamentação européia (EN 14214), e $1,9-6,0 \mathrm{~mm}^{2} \cdot \mathrm{s}^{-1}$, no caso do padrão norte-americano (ASTM D6751). 
O desempenho dos modelos propostos para o cálculo das propriedades físicas pode ser visualizada na Figura 1, a qual representa as densidades experimentais e os valores calculados através da Regra de Kay Simples (Equação 2) e pelo modelo GCVOL (Equação 4), além das viscosidades cinemáticas experimentais e as calculadas através da Regra de Kay Simples (Equação 2), Regra de Kay Modificada (Equação 3) e pelo modelo GC-UNIMOD (Equação 7).

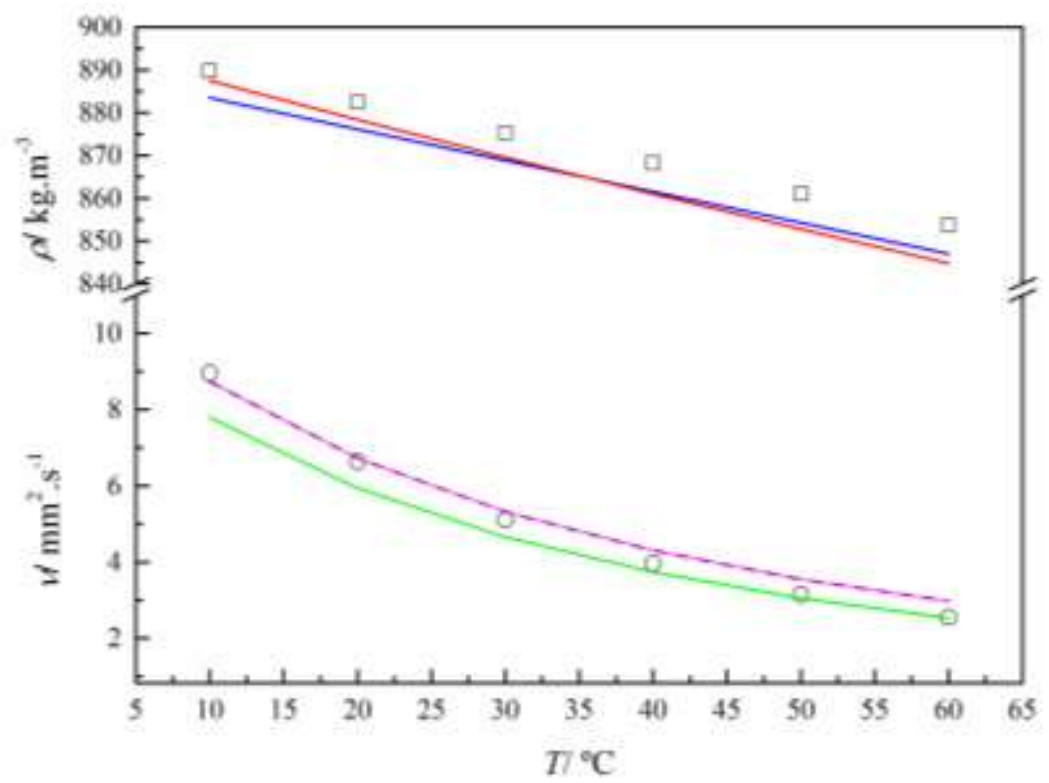

Figura 1 - Propriedades físicas de biodiesel de pinhão manso em função da temperatura $\left(T\right.$, em $\left.{ }^{\circ} \mathrm{C}\right)$ : densidade $(\rho)$, experimental $(\square)$, Regra de Kay Simples (-), GCVOL $(-)$; viscosidade $(v)$, experimental (○), Regra de Kay Simples $(-)$, Regra de Kay Modificada (- - -), GC-UNIMOD (-).

O desempenho dos modelos testados foi avaliado através do cálculo dos desvios médios relativos (DMR), calculado de acordo com a Equação 8, na qual $P$ representa a propriedade física (densidade ou viscosidade) experimental (exp) ou calculada (calc), e $N$ representa o número de dados experimentais.

$$
D M R=100\left(\sum_{i=1}^{n} \frac{\left|\mathrm{P}_{\text {calc }}-\mathrm{P}_{\text {exp }}\right|}{\mathrm{P}_{\text {exp }}} / N\right)
$$

Pode-se observar, através da Figura 1, que tanto para a densidade como para a viscosidade, os modelos de contribuição de grupos (GCVOL e GC-UNIMOD) não trazem grandes vantagens em relação aos modelos mais simples para o cálculo das propriedades físicas. Para a densidade, a Regra de Kay Simples resultou em um DMR igual a 0,76 \%, enquanto que para o GCVOL o DMR foi igual a $0,70 \%$, sendo que este último ofereceu a melhor predição das densidades apenas para as menores 


\section{9 a 22 de outubro de 2014 \\ Florianópolis/SC}

temperaturas estudadas. Para a viscosidade, os melhores resultados foram obtidos pelo modelo mais simples, a Regra de Kay, com DMR igual a 7,03 \%. A Regra de Kay Modificada e o GC-UNIMOD tiveram DMR iguais a 7,68 e 7,61 \%, respectivamente. Esses resultados comprovam que para misturas mais simples, como o biodiesel (formado basicamente por compostos de mesma natureza química, os ésteres metílicos), modelos mais complexos não são necessários.

\section{CONCLUSÃO}

O presente trabalho apresentou dados de viscosidade e densidade de biodiesel obtido a partir de uma das mais proeminentes fontes oleaginosas não comestíveis. Os dados das propriedades físicas foram utilizados para testar a capacidade preditiva de modelos simples e complexos existentes na literatura: Regra de Kay (simples e modificada), GCVOL e GC-UNIMOD. Os resultados indicaram que os modelos mais simples podem ser aplicados como uma primeira aproximação para a estimativa de dados de viscosidade e densidade, tornando-se uma importante ferramenta para a indústria produtora de biodiesel.

\section{AGRADECIMENTOS}

Os autores gostariam de agradecer à Fundação de Amparo à Pesquisa do Estado de São Paulo (FAPESP, Processo no $23203-1$ ) pelo auxílio financeiro para a realização desta pesquisa.

\section{REFERÊNCIAS}

Allen, C. A. W.; WATTS, K. C.; ACKMAN, R. G.; PEGG, M. J. Predicting the Viscosity of Biodiesel Fuels from their Fatty Acid Ester Composition. Fuel, v. 78, p. 1319-1326, 1999.

AZIAN, M. N.; KAMAL, A. A. M.; PANAU, F.; TEN, W. K. Viscosity Estimation of Triacylglycerols and of Some Vegetable Oils, based on their Triacylglycerol Composition. $J$. Am. Oil Chem. Soc., v. 78, p. 1001-1005, 2001.

CAO, W.; KNUDSE, K.; FREDENSLUND, A.; RASMUSSEN, P. Group-Contribution Viscosity Predictions of Liquid Mixtures Using UNIFAC-VLE Parameters. Ind. Eng. Chem. Res., v. 32, p. 2088-2092, 1993.

CERIANI, R.; GONÇALVES, C. B.; COUTINHO, J. A. P. Prediction of Viscosities of Fatty Compounds and Biodiesel by Group Contribution. Energy Fuels, v. 25, p. 3712-3717, 2011.

CERIANI, R; GONÇALVES, C. B.; RABELO, J.; CARUSO, M. W.; CUNHA, A. C. C.; CAVAlERI, F. W; BATISTA, E. A. C.; MEIRELlES, A. J. A. Group Contribution Model for Predicting Viscosity of Fatty Compounds. J. Chem. Eng. Data, v. 52, p. 965-972, 2007. 
CHOUDHURY, H. A.; GOSWAMI, P. P.; MALANI, R. S.; MOHOLKAR, V. S. Ultrasonic biodiesel synthesis from crude Jatropha curcas oil with heterogeneous base catalyst: Mechanistic insight and statistical optimization. Ultrasonics Sonochemistry, v. 21, p. 10501064, 2014.

ELBRO, H. S.; FREDENSLUND, A.; RASMUSSEN, P. Group Contribution Method for the Prediction of Liquid Densities as a Function of Temperature for Solvents, Oligomers, and Polymers. Ind. Eng. Chem. Res., v. 30, p. 2576-2582, 1991.

FERRARI, R. A.; OLIVEIRA, V. S.; SCABIO, A. Biodiesel de soja: taxa de conversão em ésteres etílicos, caracterização físico-química e consumo em gerador de energia. Química Nova, v.28, p. 19-23, 2005.

FREDENSLUND, A.; SORENSEN, J. M. Group Contribution Estimation Methods, in Models for Thermodynamics and Phase Equilibria Calculations, edited by S.I. Sandler, Marcel Dekker, New York, 1994.

GONÇALVES, C. B; CERIANI, R.; RABELO, J.; MAFFIA, M. C.; MEIRELLES, A. J. A. Viscosity of Fatty Mixtures: Experimental data and prediction. J. Chem. Eng. Data, v. 52, p. 2000-2006, 2007.

HARTMAN, L. Tecnologia Moderna da indústria de óleos vegetais. Fundação Centro Tropical de Pesquisas e Tecnologia de Alimentos, 330p., Campinas, 1971.

IHMELS, E. C.; GMEHLING, J. Extension and revision of the group contribution method GCVOL for the prediction of pure compound liquid densities. Ind. Eng. Chem. Res., v. 42, p. 408-412, 2002.

LARSEN, B. L.; RASMUSSEN, P.; FREDENSLUND, A. A modified UNIFAC group-contribution model for prediction of phase equilibria and heats of mixing. Ind. Eng. Chem. Res., v. 26, p. 2274-2286, 1987

PRATAS, M. J.; FREITAS, S. V. D.; OLIVEIRA, M. B.; MONTEIRO, S. C.; LIMA, A. S.; COUTINHO, J. A. P. Densities and Viscosities of Fatty Acid Methyl and Ethyl Esters. $J$. Chem. Eng. Data, v. 55, p. 3983-3990, 2010.

PRATAS, M. J.; FREITAS, S. V. D.; OLIVEIRA, M. B.; MONTEIRO, S. C.; LIMA, A. S.; COUTINHO, J. A. P. Biodiesel density: Experimental measurements and prediction models. Energy Fuels, v. 25, p. 2333-2340, 2011. 